\title{
Life-swap: how discussions around personal data can motivate desire for change
}

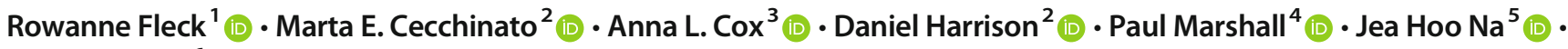 \\ Anya Skatova ${ }^{6}$
}

Received: 12 March 2019 / Accepted: 22 January 2020 / Published online: 10 February 2020

(C) The Author(s) 2020

\begin{abstract}
Personal informatics technologies support the collection of and reflection on personal data, but enabling people to learn from and act on this data is still an on-going challenge. Sharing and discussing data is one way people can learn from it, but as yet, little research explores how peer discourses around data can shape understandings and promote action. We ran 3 workshops with 5week follow-ups, giving 18 people the opportunity to swap their data and discuss it with another person. We found that these workshops helped them to recontextualise and to better understand their data, identify new strategies for changing their behaviour and motivated people to commit to changes in the future. These findings have implications for how personal informatics tools could help people identify opportunities for change and feel motivated to try out new strategies.
\end{abstract}

Keywords Data sharing $\cdot$ Personal informatics $\cdot$ Behaviour change $\cdot$ Activity tracker $\cdot$ Autographer $\cdot$ RescueTime

\section{Introduction}

Current personal informatics (PI) technologies provide us with vast amounts of data about many aspects of our personal, professional and social lives. Reflecting on that data in order to gain actionable knowledge about how to become fitter, happier and more productive is believed to be one way of improving our quality of life [1]. Personal data might help people become aware of issues to do with managing different aspects of their lives. There are now several applications and

Rowanne Fleck

R.fleck@cs.bham.ac.uk

1 School of Computer Science, University of Birmingham, Edgbaston, Birmingham B15 2TT, UK

2 Computer and Information Sciences Department, Northumbria University, 2 Ellison Place, Newcastle upon Tyne NE1 8ST, UK

3 UCL Interaction Centre, University College London, 2nd floor 66-72 Gower Street, London WC1E 6BT, UK

4 Department of Computer Science, Merchant Venturers Building, Woodland Road, Clifton, Bristol, UK

5 Manchester School of Art, Manchester Metropolitan University, 1.02 Righton Building, Cavendish Street, Manchester M15 6BG, UK

6 School of Psychological Science, University of Bristol, 12a, Priory Road, Bristol BS8 1TU, UK devices on the market that monitor and measure almost any aspect of our life (e.g., Fitbits, weight loss apps, softwarelogging tools) and have led us to a data-driven life. Recently, tech giants such as Apple and Google have announced new monitoring features that allow users to take control of their digital habits and better spend their time [2]. People collect data with these technologies with multiple intentions, including: to reflect on their routines, learn about themselves and potentially change their behaviour. However, despite much recent work in this field, supporting people in making sense of their personal data is still an on-going challenge [3], in particular, translating the information included in this data into actionable knowledge [1].

Sharing data with others is one approach to address this challenge and can potentially lead to and support changes in behaviour [4-6]. Understanding how our own data compares to others has been identified as an important part of learning from PI data [7]. However, despite a number of authors describing PI data as a social practice $[8,9]$, current PI tools tend to limit 'data sharing' to social media or in-app leaderboards. It is less understood how discussing and sharing our personal data with others can support meaningful reflection on it, or how this could be best supported. Therefore we are potentially missing out on opportunities to exploit social practices in different contexts to support understanding.

Our aim was to investigate how sharing data in a face-toface context might influence understanding and promote 
action-taking. We present findings from three 'Life-Swap' workshops, where new and existing PI users could share personal data, in order to collectively understand more about their own work-life balance (WLB) and ways they might change it for the better. The workshops involved participants bringing and discussing data they had collected, either with a softwarelogging tool that captured their digital routines; a wearable digital camera that automatically captured a series of firstperson images of their daily routines; or activity trackers. After 5 weeks, we followed-up with our participants to understand whether any insights gained and intentions to change were translated into actions. Motivated people to commit to their data in this context helped participants to recontextualise and better understand their data, identify new strategies for improving aspects of their WLB, and motivated them to commit to changes in the future. This paper makes the following contributions

- We extend our understanding of how sharing and discussing different types of personal data can help the sensemaking process of PI tools.

- We discuss how this leads to intentions to change and action.

- We offer implications for how PI tools could be used to raise opportunities for change.

\section{Related research}

Many PI applications and tools enable users to share the data they log and collect with others in a variety of ways, for example through generating tweets, posting to social media sites, or via in-application comparison tools. The benefits and issues associated with sharing personal data in these ways have been explored in domains such as fitness and health. However, very little previous work talks about one-to-one peer sharing of, or discussion around, personal data.

\subsection{Sharing personal data}

The literature has identified many reasons why people are either motivated (or compelled) to share such data logs. For example, people might wish to share data to get some kind of information from their audiences, like recommendations or advice on something different to try; in order to receive emotional support; as a source of external motivation, or to motivate an audience; and as an impression management device [See [8] for a review]. People may also be compelled to share data as a means for their behaviour or health to be monitored, for example sharing driving logs with car insurance companies, or being required share health data with a health-care professional [6].
Less discussed are the benefits that people get from other people's data. Ploderer et al. [10] do discuss the value of 'social traces' or traces or patterns of other users which can be anonymous (e.g., usage statistics) or created by known others (e.g., celebrities). Such traces of other people's data can raise awareness and discussion of issues (such as air quality), provide something to follow, indicate 'norms' (i.e., expectations for appropriate behaviour within a social setting [11]), which can prompt people to adjust their own behaviour to better match these [12], and to encourage comparison and competition which can motivate change, but can also inhibit it [13]. Therefore, there are potentially benefits to both those who share data and to those who can see and explore that shared data.

Whilst there is huge interest in encouraging people to share data online, via apps, and social networks, there are also many issues surrounding this. People might be reluctant to broadcast this data to a wide audience despite the potential benefits to themselves, and there are tensions between privacy and the usefulness of the shared data to others [10]. For example, Epstein et al. [8] found that automatically-generated content shared online through in-app features (e.g., "today I ran 5.3km") was considered boring compared to online shared content where users provided more details about the context of their data.

As we move towards big (personal) data, privacy concerns become more prevalent and revolve around a whole suite of issues such as who has access to that data, how would this data be used, what control does the user have over their data, etc. Often users have to make a decision around the trade-off between the sharing of their data - even for the sake of gaining more insight - and retaining privacy, something that Tolmie et al. [14] refer to as privacy management. Considering ways to facilitate users to share and discuss data offline might become an interesting alternative to that trade-off decision, whilst allowing users to retain more control over their data, who has access to it and for what purpose.

We argue that there is value in sharing this data in one-toone or private settings, e.g. small groups of people in workplaces, community groups, schools or small and secured online groups, where some of these issues become less relevant and it is possible for sharers to become involved in richer discussions about the shared data. To this point, Elsden et al. [1] created a speculative environment to explore the social life of data and understand how users might decide to share their personal data with someone else in a one-to-one private setting. Whilst their findings focus primarily on how the data becomes a ticket to talk and a way of self-expression in a simulated speed-dating event, the authors point towards the importance of placing data into context, and discussions having some consequence for participants. They also call for more explorations around how to socialize around one's data.

Fleck and Fitzpatrick [4] looked at how images captured by a wearable camera supported teachers' reflective discussions of their lessons. During paired discussions, the images 
allowed teachers to see things they had missed at the time, and the conversations were vital in supporting the pairs in describing, explaining, questioning and reinterpreting the events of the lesson, sometimes leading to new insights and intentions to change future practice. They suggest that such discussions benefit both parties, as the observer can help the sharer interpret and better understand their data, and the experience of seeing and discussing another person's data leads to sharing experiences and insights into own behaviour too. However, this study mostly involved discussion of just one person's recording, rather than both parties bringing their own data.

In another study, Graham et al. [15] asked participants to share personal data (including physical activity data, food, teaching effectiveness, screen time, etc.) they had collected over a period of 4-6 weeks. In this case, both parties shared data with each other remotely and asynchronously. Participants were asked to comment on each other's data, and then respond to the comments of others on their own data. Despite the different format, they found similar patterns to Fleck and Fitzpatrick [4] in the ways that participants supported each other's interpretation and reflection on the data, which they claim led to 'actionable' insights. However, they did not follow-up with their participants to understand whether intentions to change translated into valuable actions.

\subsection{Aim}

Our motivation for doing this work was to explore ways that conversations around different kinds of personal data can support collaborative reflection, not just on the data itself, but also on experience around a certain issue, and how this can motivate desire for change. We are particularly interested in the ways that such conversations around data can provide an insight or lens into another's life, that can help us understand our own data, and our own life, better. Given that previous research has found sharing and discussion around data to be an effective means to promote reflection and learning from data, we were interested to see if sharing personal data, which in some way captured work and non-work routines, might support discussions and reflection on issues of work routines vs. home/personal routines; work breaks and recovery; wellbeing at work; and managing boundaries between work and non-work, and lead to desire for change. Therefore, in extending work of Graham et al. [15] and Fleck and Fitzpatrick [4], we arranged a series of data life-swap sessions where participants were invited to come, share, and discuss data about their work and non-work practices and how they might influence their WLB. In this paper, we focus on how the process of data life-swap supported their understanding of the data, their own WLB practices, and their exploration of ideas for how they might change these for the better.

\section{Method}

\subsection{Participants}

A total of 18 participants took part across 3 workshops (see Table 1). Participants were paid $£ 15$ in vouchers to attend, plus an additional $£ 10$ on completion of the follow-up survey 5 weeks later.

Six female participants, all $\mathrm{PhD}$ students between the ages of 23 and 29, took part in the RescueTime Workshop. They were recruited via an advert in the post-graduate research hub of a UK university asking for students who were interested in discussing their work-life balance and productivity.

Seven female and one male participant between the ages of 25 to 32 took part in the Autographer Workshop. Four of these participants were employed and worked in an office environment, whilst four were PhD students. These participants were recruited from a pool of participants from an earlier study who had already collected a week's worth of Autographer data during a working week approximately 1 year before the current study [16].

Three male and one female participant, aged 22 to 33, took part in the activity tracker workshop. They were recruited via posters at the research team's universities and via social media. Two were office workers and two were Masters' students.

\subsection{Design of the Workshops}

Three workshops were held in three locations in the UK. At each workshop, participants were asked to bring along a different set of data that could offer them insight into aspects of their work/non-work routines.

Workshop 1 focused on data from RescueTime (https:// www.rescuetime.com), a commercially available software which automatically logs the applications participants' run on their devices, and provides various visualisations with which to explore this data (Fig. 1). They include bar charts

Table 1 Summary of workshops

\begin{tabular}{llll}
\hline & Collection tool & Familiarity with data & Participants \\
\hline WS1 & RescueTime & Recent novel data & $6 \times \mathrm{PhD}(\mathrm{R} 1-6)$ \\
WS2 & Autographer & Old familiar data & $4 \times \mathrm{PhD}, 4 \times$ office workers (A7-14) \\
WS3 & Activity Trackers & Old and recent familiar data & $2 \times \mathrm{MSc}, 2 \times$ office workers (T15-18) \\
\hline
\end{tabular}


$6 \mathrm{~h} 46 \mathrm{~m}$ Logged this day

$6.8 \mathrm{~h}$ more than the day before

time by hour

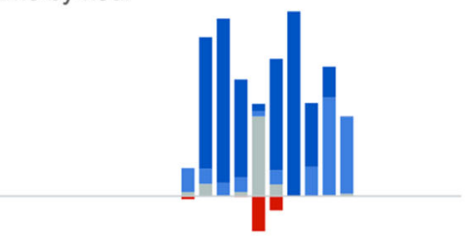

12AM 3AM 6AM 9AM 12PM 3PM 6PM 9PM productivity pulse

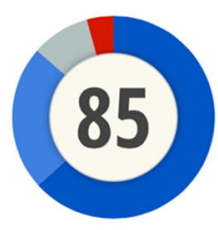

- $70 \%$ from day before

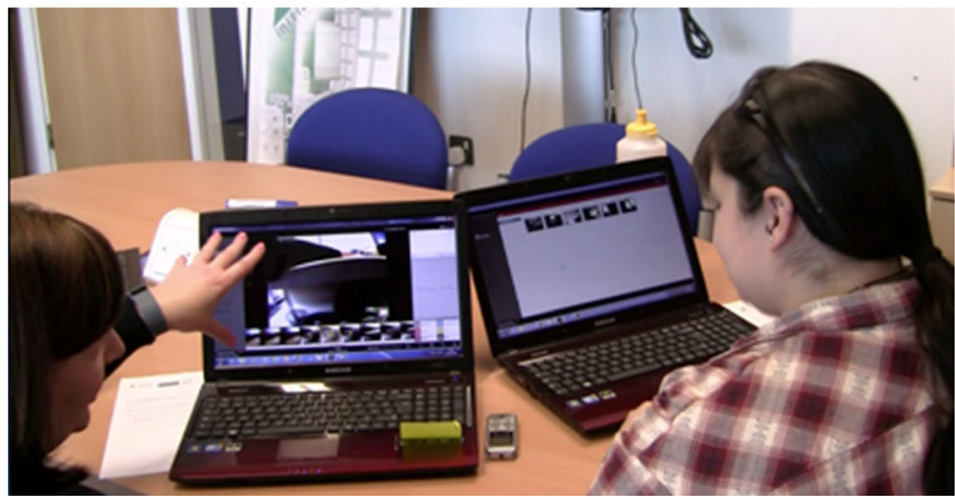

Fig. 1 Example of RescueTime data (left) and conversation around Autographer data (right)

of applications used at various times of the day and over time, and a 'productivity pulse' calculated by comparing the amount of time spent on productive tasks (like writing and communication tasks) vs. unproductive tasks (like checking social media sites). Users can configure which applications the system considers productive or unproductive themselves.

Workshop 2 focused on data from Autographer cameras (http://autographer.com), a wearable digital camera, worn around the neck like a pendant, that automatically takes approximately 2-3 images a minute. Images can then be played back on a PC using viewing software, enabling participants to view their days as a sped-up, first person perspective movie (Fig. 1).

Workshop 3 focused on data collected from the participants' choice of activity tracker. Some participants brought data from wrist-worn activity trackers, whilst others collected data via smart-phone sensors and applications. We felt that this data could reveal participants' routines i.e., where they were and what they were doing at various times, that would enable them to reflect on aspects of their work/ non-work routines.

\subsection{Procedure}

\subsubsection{Prior to the workshop}

Before attending one of the three workshops, participants provided basic demographic information and completed a deviceuse questionnaire to find out about the technologies they usually use (see Fig. 2). Information about participants' work/ subject of study was used to organise participants into conversational pairs at the workshop, and their device use to offer them the correct support to install research software.

Before Workshop 1 (WS1), participants were asked to install RescueTime on all their work computers, and Android phone if they had one, for at least 2 weeks prior to attending the workshop. None of the participants had previously collected data like this before the study, therefore it was recently collected data, and novel for participants.

Participants who attended Workshop 2 (WS2) were provided with data they had collected data during the previous study [16]. It had been previously reviewed with an experimenter, though participants had not seen it since and no longer had

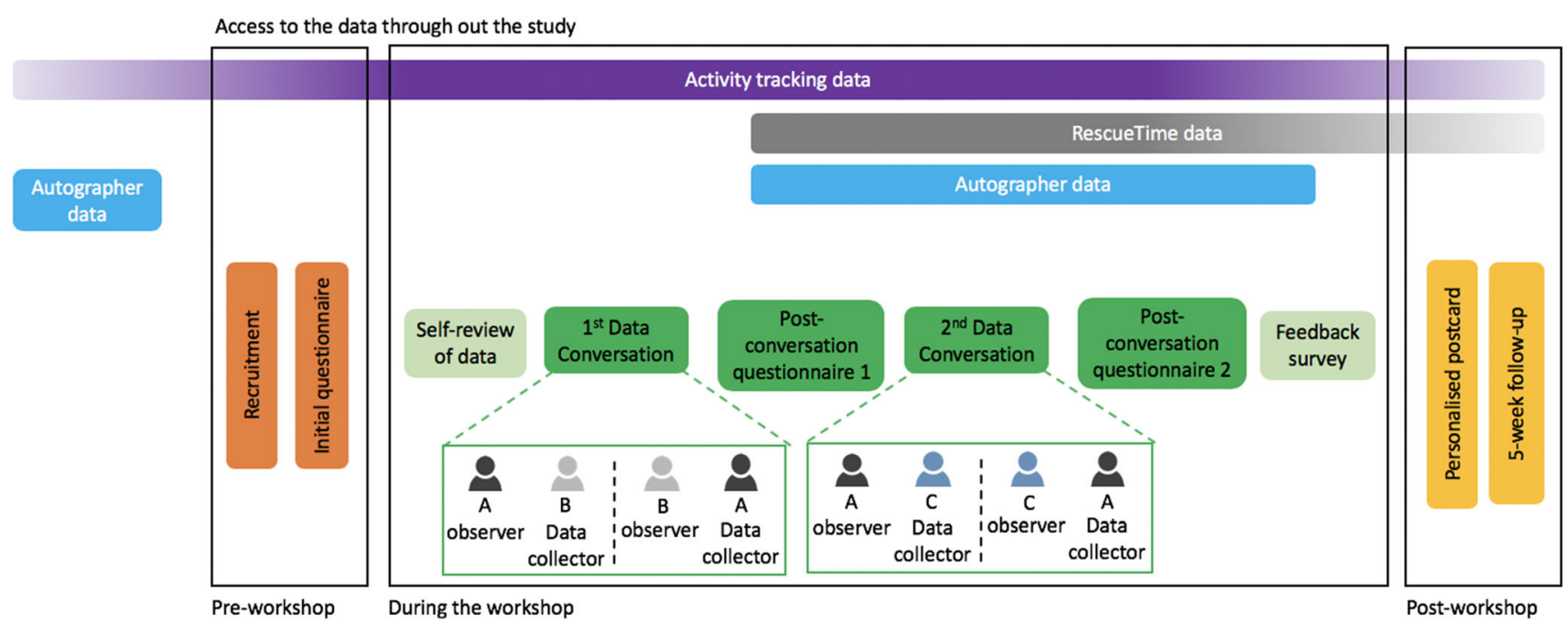

Fig. 2 Diagram illustrating the study timeline from personal data-collection, workshop session procedure to 5-week follow up 
access to the collection tool. Therefore, this data was about a year old, and familiar to participants.

Workshop 3 (WS3) participants were asked to bring along their own activity tracker data. Therefore they were familiar with this data, and all but one had been collecting it for over 2 years.

\subsubsection{During the workshop}

All workshops lasted between 2.5 and $3 \mathrm{~h}$. Apart from the kind of data discussed; all three workshops followed the same structure. An attempt was made to create a friendly and relaxed environment at the workshops to facilitate conversations around personal data. On arrival to the workshop, participants were offered refreshments, then given approximately $10 \mathrm{~min}$ to go through their data themselves at which point they were invited to edit/delete anything they would not feel comfortable sharing with others in the room. They were then organised into conversational pairs and given guidelines on how to proceed with their data conversations. Participants took part in two conversations with two different participants. As far as possible, they were asked to speak with someone with a similar job/area of study in the first conversation, and a different one in the second conversation. (see Fig. 2).

\subsubsection{Data conversations}

1st conversation Participants were given four conversational prompts on themes related to work-life balance to encourage discussion and reflection on these topics. The prompts were: work routines vs. home/personal routines; work breaks and recovery; wellbeing at work; and managing boundaries between work and non-work. They were asked to select 1 or 2 days, for which they had data, to discuss with their partner. We suggested that one person (taking the 'data-collector role') go through their days first, going through their data and talking about their day/data with the other participant (taking the 'observer role'), before swapping roles. Conversations lasted as long as participants wanted them to up to a limit of $45 \mathrm{~min}$, and they were encouraged to go back to and use the data in any way they wanted to support their discussion. After the conversation, participants individually filled in a post-conversation questionnaire, which asked them to note what they learned from the conversation, and things that they might like to try/behaviours they might like to change following the workshop.

2nd conversation After a short refreshment break, participants were reorganised by the facilitators into their second conversation pairs, and second conversations proceeded in exactly the same way as the first but with a new partner. On completion of the second post-conversation questionnaire, the workshop finished with participants filling in the final workshop questionnaire where they provided information about their experience of the workshop. All conversations were video and audio recorded to support later analysis.

\subsubsection{Five-week follow-up}

Approximately 3 weeks after the workshop, participants were sent personalised 'Life-swap' postcards reminding them of any changes they had said they would like to make in the post-conversation surveys. These were written in their own words (see Fig. 3). Two weeks after this, they were asked to complete a final online questionnaire where we asked them to tell us whether they made any of the changes they had intended to, or any other changes related to the themes of the workshop, and what prompted these.

\subsection{Analysis}

Each of the 18 participants took part in two conversations, resulting in a total of 18 conversations ( 2 people per conversation, 2 conversations per person) across the three workshops, all of which were transcribed. Coding of conversation transcripts was then conducted using both inductive and deductive techniques for thematic analysis. Initial codes around
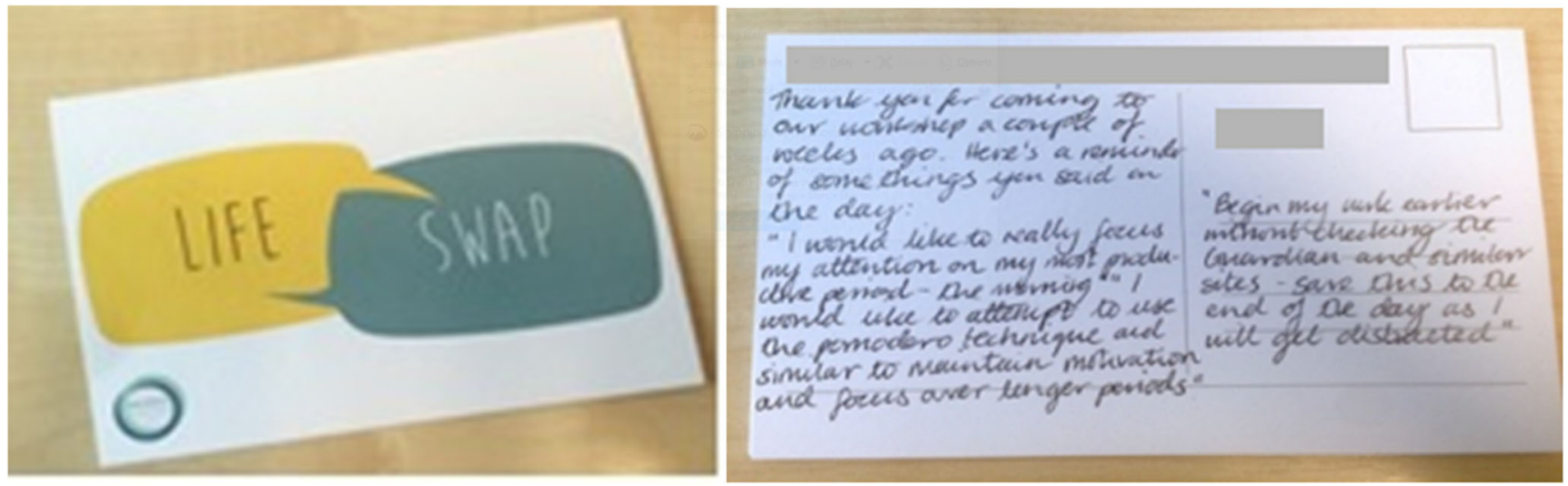

Fig. 3 Post-cards sent to participants 2 weeks after the study 
the themes of the research were inductively defined independently by two members of the research team through a process of open coding of two randomly selected conversations. These initial codes were then compared and discussed by the research team and a total of 30 non-mutually exclusive categories under two major themes and six minor themes (conversation content -issues/problems, strategy/tactics, session talk; life-swap process-reflection on data, sharing experiences, intention to change) were agreed on and used to deductively code the remaining data. Finally, written answers to the questionnaires were analysed to see what participants took away as intentions to change and to understand how these related to the conversations. These were also compared to actual changes participants reported they made 5 weeks later.

\section{Findings}

Although conversations unfolded in a natural way moving iteratively through stages, most roughly followed this pattern: (1) the data collector started by describing and explaining their data; (2) the observer and the data collector then entered a period of collaborative interpretation of the data including a back and forth of asking for more information, further description and explanation, justifying, comparing and contrasting and empathising with each other; (3) they swapped roles and repeated 1 and 2 (the other participant shared their data); and (4) they went through the conversational prompt sheet and discussed any points they had not already covered.

Through this process, more than just the data was shared: participants shared, compared and contrasted not only their data, but their experiences of issues that the data revealed, and some of the strategies and tactics that they employ to manage this and overcome difficulties they have had. This led to a few instances of participants gaining new insights. All but one participant generated intentions to change their behaviour, which the majority acted on in the weeks following the workshops.

We now describe in more detail how the conversations unfolded and led to actionable knowledge in the form of intentions to change their behaviour. Firstly, we illuminate how participants shared their data in the conversations we observed; then we discuss in more detail how they compared and contrasted experiences, strategies and tactics; and finally, we will describe how this lead to action.

\subsection{Sharing data}

Although participants were free to discuss whatever they liked as they looked through the data, the theme of the workshops was WLB, and in all sessions they were provided with the same discussion prompts on this theme. Therefore, we did observe much discussion around these topics, especially in the RescueTime and Autographer conversations. In Activity Tracker discussions, there was more emphasis in discussion on activity aspects of work-life-balance, and about the tracker technologies themselves.

\subsubsection{Description and explanation}

Conversations around all three datasets began with the first data collector describing their activities as evidenced by the data, or describing their tracking technology. This initial description also involved explaining the data and putting it into context for the other person. This included explanations of how typical or representative they felt the data was of their normal behaviour, sometimes questioning the validity of what the data seemed to show. Whilst conversations started off with this type of description and explanation of data, it continued throughout the conversation though in a slightly different way around the different types of data.

Participants discussing Autographer and RescueTime data spent more time - compared to those discussing activity tracking data - describing what the data showed. This included talking about their working routines, e.g. when they started work, what work activities they did, what other non-work activities they did, things that distracted them during the day and how they took breaks.

In Autographer cases it took time for participants to play through their images sequentially, and they described the day's events in detail, step by step, as they went through, leading to proportionally more description of the data in these conversations, and many short statements of what was going on:

\section{"A14: Oh here [...], someone's phoned me and I've gone out the office into a stairwell to take a [call]".}

There were also much longer descriptions and explanations:

"A9: Yes we often did that [...] we had training sessions because we're quite a, this team now had only been together for a year. So we're still getting quite established as a team, people were picking up new responsibilities, so we often had like training sessions at her desk with her".

RescueTime data prompted more explanation from participants, who would both describe what the data showed, and attempt to interpret or explain that for their partner:

"R6: So, I did a bit of work in the afternoon, because I wanted to get something out of the way, I needed to just update a chapter draft, it was just some sort of mechanical task, and I wanted to get it out of the way before the 
new week starts, but the rest of the day was just having fun I think".

This also included indicating to their partner how representative they felt the data was of their usual routine, e.g.:

"R5: I usually use Telegram and WhatsApp a lot. Because, all the time my sister and my Mum will be messaging me to check how I am and I'll be checking to see how they are as well".

However, in conversations around Activity tracker data there was more focus on the tracker technology itself - including how it worked, what the data allowed you to see, as well as how they used the technology or the data:

"T15: I use a Microsoft band 2, it has UV sensor so it can see sunlight, it has heart-rate tracking, skin conductance... you are familiar with this?

T16: no! It's new to me.

T15: and GPS, and accelerometer. So I usually use this when I go out the house, usually when in the house I do not... it's still quite bulky so it's a bit inconvenient for me... so I use it mainly to [starts to look at display on phone] track my days, so... [both looking at phone display]".

They did also describe what they were doing on individual days, but only briefly, and most often early in each participant's turn as data collector. Also, more so than in other conversations, Activity Tracker participants described general patterns of behaviour, showing the observer an overview of wider time periods of data, then zooming in to explain extreme values, e.g.:

"T18: On June 28th, I went to [anonymised location] by walking. And the market is a pub and a music market. So I walked there and took, uh... And you can see from the data board some, the largest number is last Sunday. Let me see. I went shopping with my sister, and I spent a whole afternoon. So the number of steps was 8446. And the longest distance is $5 \mathrm{~km}$. I think this is the most... the lowest is last week".

\subsubsection{Questioning the data}

Participants also went further than highlighting the representativeness of the data they were sharing with their partners, and questioned the validity of data - including justification or explanation of what the data was/was not showing, sometimes with reference to limitations of the data collection.
In Autographer discussions, misrepresentation was discussed as being due to not being able to wear the camera at all times (due to privacy concerns), the camera simply not capturing everything or finding it hard to get an accurate sense of proportion of time spent of various activities, for example:

"A14: I don't know why I have started it at 10.22 because I usually get in a bit earlier, but I must have forgotten to turn it on".

With RescueTime data, as there was more ambiguity and so interpretation needed. Participants suggested limitations of the technology as an explanation for surprising observations, for example, being unconvinced about how it had chosen to categorise activities or that it was not really capturing what it claimed:

R3: "But, the thing is, I don't tend to use a lot of referencing stuff, like I don't tend to go on databases and stuff a lot. I tend to work a lot on Microsoft Word. So, I don't know, I think the flaw's in the software, I think to be honest. It doesn't always pick up what you're most productive at".

Whilst participants discussing activity tracker data did not seem to challenge the validity of the data they had collected, they did discuss limitations in their ability to collect the data they would like to (e.g., not being able to wear trackers during certain activities like swimming or dance, or battery limitations).

\subsection{Collaborative interpretation of data}

However, this was not a one-way process: the observer did not only act as an audience necessitating explanation of events, but actively prompted for more information to aid their understanding and gain more insight into the collector's routines. They also brought their own perspectives and experiences to the conversation. Therefore, after the collector had begun describing and explaining its data, the conversation moved into a back-andforth stage where the collector's on-going description and the data itself prompted questions from the observer leading to further discussion and explanation, justifications, comparing and contrasting of data and experiences and empathising with each other. This often took the conversation well beyond the data.

Around all data, the collector's on-going description prompted questions from the observer leading to further discussion. In Autographer cases, especially the observer would see something in the data which prompted them to ask for more information, for example:

"A14: Were you drinking there? Is that actually like a bottle?

A12: Yes it's like one of our, like it's one of our promos. A14: Okay. 
A12: Yes I drink like two of those a day.

A14: Just water?

A12: Yes just water, or water sometimes with squash or just water".

RescueTime data prompted questions from both parties who would collaboratively try to piece together what the data represented, as in this example where the collector asks the observer to help them interpret the data, leading to a more general discussion on how they are distracted by social media:

“R3: What else have I got? Communication, what's
that?
R1: So e-mail...
R3: E-mail, e-mail, yes. Yes that's it, that's all that I do on
my, either on my laptop or my PC, is mainly work, so it
does not, it's not logged all my phone activities and
slacking. You've got quite a lot of social networking;
you have got like good social networking.
R1: Yes that's the thing, good, there's no such thing. Yes,
you can see it's just Facebook, so it's just...
R3: Do you tend to leave that like running in the
background?
R1: Yes, that's the problem, and then if I get a message
from someone then I'm like ooh a message and put my
reading away and just end up, yes, going on there”.

This did happen in Autographer and Activity Tracker discussions, but less frequently.

\subsection{Comparing and contrasting experiences}

As described above, the process of sharing data involved discussion and description that went beyond discussing just what the data was showing. As participants compared and contrasted their data, they naturally also compared and contrasted the experiences behind the data with each other. The conversational prompt sheet also triggered some of these comparisons.

\subsubsection{Work-life balance routines, strategies and tactics}

In Autographer and RescueTime discussions, this included discussions about their working and other daily routines, and problems and issues they had. They also compared and contrasted strategies or tactics they use to manage their working routines, and that they have attempted to use to try to overcome difficulties they have identified e.g.:

"A10: [...] I suppose move, moving around... I often yes, I had people turning up at my desk, so you know, like, that's it then, game over.
A8: Yes, I make more of an effort to move round now, um, when I am working, either at home or in the office, to not be looking at screens, I have started having my break, like when I have lunch, I have started not sitting in front of my computer when I do it, because then it ends up not feeling like a break at all and I just keep working, so I have started doing that, or meeting for lunch".

However, in Activity Tracker discussions, sharing WLB strategies tended to happen towards the end of conversations in response to the conversation prompts we gave them. These were generally focused on ways to fit activity into their daily routine.

\subsubsection{Technology strategies and tactics}

In addition to discussing issues around the topic of the workshop, participants also discussed their use of the tools used to collect the data. For example, participants who discussed activity tracker data were all shared and compared how they used their trackers. In one discussion between two keen wrist-worn activity tracker users (Participants T15 and T16), this made up a large part of the conversation, for example:

"T15: The other thing I like is actually, it can measure your heart-rate, so some blood or pulse kind of thing [takes off his watch to demonstrate].

T16: yeah, this one can do that as well. But the light does not go on when you take it, so it has that as well. [takes off his watch to demonstrate].

T15: But you do not use the data?

T16: um, I... no. I... it's interesting, but not really that much, no! [laughs]".

Similar discussions occurred, though less frequently, in RescueTime conversations, yet not in the Autographer discussions. For RescueTime, participants talked about and shared tips on how they had adapted their use of the technology to better support them in understanding their working routines, and compared ideas on how it could be useful to them. For example:

"R3: It's logged Design and Composition a lot, and I think that's mainly just like Word and stuff.

R4: Yes, I've changed my Word, I've put it under Referencing and Learning because I could not find it and then I saw it under Design and Composition and I thought, I'm never going to remember that, so I put it under Referencing and Learning.

R3: That's actually a good idea. I think some of the categories are a bit...

R4: They're very odd". 


\subsection{New insights and intentions to change}

In order to understand how participants gained actionable knowledge through the process of looking at and discussing their data, we traced through the conversations and postconversation questionnaires to see what triggered new insights and intentions to change around the different data sets. Not all the things we had coded as new insights, or that participants had explicitly stated during discussions that they would like to try, made it to the post-conversation questionnaires. And although not everything they stated that they wanted to change in the questionnaires was explicitly said during discussions, it was clear how they had arisen from discussions.

\subsubsection{RescueTime: Recent and novel data}

More new insights and explicit statements of intentions to change behaviour were evident in RescueTime discussions than around the other two data sets. Insights in RescueTime discussions were triggered by looking at and discussing the data (for example noticing how much time was spent looking at social media or checking email, noticing at what time of day they were more or less productive, and realising that breaks from work were often spent looking at screens). They were also triggered as a result of sharing experiences with each other, for example R1 became aware of how little time she took off when she compared her week with R3. R1: "I realised that I didn't take a lot of time off from my work, as I work another job over the weekend.", which led to the intention R1: "I want to review the time-off I take from my work, specifically during weekends". Similarly, whilst some ideas for things to change came from participants' observations from the data, many ideas for changes to make came out in the process of discussion around the data, and sharing and comparing ideas and tips for things to try. Additionally there was evidence of participants further exploring ideas raised in the first conversation in their discussions with a new partner in their second conversation, and of ideas discussed in the first conversations noted as intentions to change only in the questionnaire after their second conversation. For example, R4 decided to "Begin my work earlier without checking The Guardian and similar sites - save this to the end of the day as I will get distracted". Following a discussion with R5 where she told her she always left checking the news until the end of the day because it upset her (though she noted this only after her next conversation).

\subsubsection{Autographer: Old and familiar data}

As Autographer participants had already seen, discussed with an experimenter, and had time to act on insights gained from the data they had collected, there were fewer 'new' insights that arose in these conversations from looking at the data alone. Instead participants discussed insights they had had in the past and changes they had already made. These included changes made in working routines (e.g., when they start work, having started using Pomodoro technique) and in particular how and when they take breaks. However, this was the first time participants had compared their data with someone else, and as with RescueTime, new insights, observations, and ideas for ways to change behaviour were raised and shared between participants and conversations. Breaks from work, and how other people took breaks, was raised a number of times leading to ideas for changing these (e.g., eating away from the desk, having a more stable or regular 'break' routine). They were interested and surprised at other people's routines - in some cases they stated they would not be able to work as the other person did (either because their work would not allow it, or it would not suit their personality), but in some cases, they got ideas from the other person on how to manage their time differently. For example, one participant decided to try to work in cafés rather than at home when not in the office as their partner found this helped limit 'homebased' distractions. One participant had started to successfully use the Pomodoro technique recently, and one of her partners and another participant left these sessions intending to try it.

\subsubsection{Activity tracker: Old and recent familiar data}

In contrast to the other 2 groups, participants who tracked activity tracker data had in all cases looked at their data before, and in most cases ( 3 out of 4 ) been tracking and looking at their data for more than 2 years. Therefore, they were very familiar with it and were intending to continue tracking and monitoring. Because of this, there was only one seemingly new insight gained just from looking at the data, though as in Autographer discussions, prior observations were discussed. These included becoming aware of what kind of situations caused stress and how levels of exercise and their weight vary considerably in relation to times of year/ changes in working patterns. Instead, ideas for change came from the process of comparing and contrasting their own experiences with others: for example, one participant told his partner about how he uses his heart rate to monitor his stress levels in various situations leading the partner to say he wanted to monitor his heart rate more and respond to his mental wellbeing and stress levels. Others were inspired to try and adopt each other's practices too, for example monitoring weight, (T15: "He was tracking much more data which I found really useful. For e.g., weight") wearing trackers more often, having a better charging routine and fitting more exercise into their daily routines.

\subsubsection{Five-week follow-up}

In order to understand whether any of our participants' intentions to change behaviour led to action, we conducted a follow-up survey 5 weeks after the workshop. All 18 
participants responded to our enquiries, 14 of whom (6/6 RescueTime participants, 5/8 Autographer participants and $3 / 4$ activity tracker participants) reported having made changes to their work life routines, use of technology and/or their levels of physical activity as a result of participation in the workshop (see Table 2). The RescueTime conversations lead to the most successful changes. However, one participant (R3) forgot a change they said they would try, and one switched from intending to take breaks away from her desk to "making lists for everyday so I know I have achieved something" (which did follow from a conversation she had had at the workshop). Though two of the participants were rather vague about their goals and how they had achieved them (e.g., R5: "to be productive and avoid procrastination"), all reported that these changes had had a positive impact on them (e.g., R5: "I feel better and more productive").

Fewer of the participants from the Autographer workshop reported attempting to make their intended changes and there was more discrepancy in this group between what they stated they intended to change at the end of the workshop and what they remembered they had intended to change 5 weeks later. One participant who did not make any changes had not intended to. Of the five that did make changes, four remembered additional intentions to those stated in the questionnaires after the workshop (e.g., being more active generally, limiting social media time), though these were topics that had come up in their conversations. They also reported mixed feelings about the success of these changes.

Finally all activity tracker participants had intended to make changes to their levels of physical activity and all but one their work-routines. Overall their remembered intentions were very similar to those they came out of the workshop with, although one participant had turned general intentions into more concrete goals. One participant cited unusual work circumstances for hampering efforts to make intended changes, and one reported only partial success in increasing his levels of physical activity, but otherwise participants claimed success in making their intended changes. These included taking less work home at the weekend, working more regular hours and fitting in more regular exercise.

Therefore, our data suggests participants did try to put into practice some of the changes they stated they intended to during the workshop. Although not everyone tried everything, and some were not overly positive about their attempts, most participants reported positive outcomes from these changes.

\section{Discussion}

Our 18 data life-swap conversations have enabled us to unpack how sharing different types of personal data can help the sensemaking process of PI tools. More specifically, they have allowed us to detail how participants learned from their data and each other in a way that generated actionable knowledge and lead to change.

\subsection{Value of sharing and discussing own and others' data}

The discussions highlighted the value of always having both data collector and observer's points of view: throughout conversations both were involved in interpreting what the data was showing, including questioning the validity of the data and the accuracy of the tools used to collect it. The collector benefitted from having support in making sense of what their own data was really saying, and received positive support from the observer. The observer also found benefit - as evidenced by the questions asked to gain more information about what was going on around the data, and about the behaviours and routines surrounding it.

\subsubsection{Importance of recontextualising data through sharing}

Very apparent from our conversations, therefore, was not only what the data showed, but what it did not. For the other person to make sense of it, participants needed to describe the context. This included describing the context in which the data was collected, explaining how accurate or representative the data or what it seemed to be showing was of their own experience, and often justifying why they behaved as they did or even why the data may appear to be showing something other than the 'truth'. Reflecting on one's own experience and data in this way has been shown to lead to new insights [17, 18], and drawing correct conclusions from data in critical situations such as health decision making [19]. But in addition, it was the richness of these descriptions and explanations, and the detail beyond the data, that lead to the comparing and
Table 2 Types of changes and their frequency amongst participants

\begin{tabular}{llll}
\hline Workshop (total participants) & $\begin{array}{l}\text { No of participants who made changes/number of participants who intended to } \\
\text { make changes }\end{array}$ & & \\
\cline { 2 - 4 } & work-life routines & use of devices & physical activity \\
\hline RescueTime $(N=6)$ & $4 / 4$ & $2 / 2$ & $1 / 1$ \\
Autographer $(N=8)$ & $5 / 7$ & $2 / 3$ & $4 / 4$ \\
Activity Tracker $(N=4)$ & $3 / 4$ & $1 / 1$ & $3 / 4$ \\
\hline
\end{tabular}


contrasting of experiences, understanding of own data in the context of others, and the sharing of tips and strategies. This was what ultimately generated the ideas that lead to the intentions for change that were then actioned.

Data-logging applications have supported sharing for some time; this is often accomplished through aggregated data or leader boards. Since conducting this research, RescueTime is piloting new features to enable people to compare their own data with other people 'like them'. Whilst there will clearly be some value in this in enabling people to understand themselves in relation to others, our conversations highlight just how much will still be missing from this data, limiting the validity of the comparisons that can be made. Additionally, Tolmie et al. [14] talked about how focusing solely on decontextualised data could have serious consequences due to misrepresentations of people's lives after getting some surprising accounts from their participants of what their personal data showed. Similarly, attempts to compare oneself to decontextualised, anonymous data could be dangerous unless enough context is provided, especially as previous research has suggested that 'data doubles' can change the way people reflect on themselves [20]. This is especially important when considering health data or levels of physical activity [19].

\subsection{Datasets and sharing environments affect insights}

Whilst our workshops all followed the same structure and had the same conversational prompts around work-life balance, there were a number of differences between workshops. The types of data, the tools used to collect it, the timescales of data collection, the levels of granularity and the levels of familiarity with the data and the tools were different. One could argue that these differences hinder any comparison of the discussions and reflections between datasets, but we emphasise how this has led to valuable observations, which we discuss below, which have implications for contextualising existing PI findings and opens up future research directions.

Nature of data Both the nature of data and what it showed/ emphasised, and the conversational prompts, directed participants' conversations. The Autographer and RescueTime data lent themselves well to the prompt themes (work routines vs. home/ personal routines; work breaks and recovery; wellbeing at work; and managing boundaries between work and non-work) and data discussions were mostly on these topics throughout. Activity tracker data however did not lend itself so directly - resulting in a disjoint between discussion on what the activity tracker data did show and the workshop themes. However, in all activity tracker cases, participants discussed the data in terms of the workshop themes at some point. This highlights the potential to direct conversation around data to facilitate reflection on specific topics, but that further research is needed to explore how best to do this.
Granularity and timescales Autographer data was very detailed, with several pictures taken per minute, participants only had a week's worth of data to choose from, and typically only discussed 1 or 2 days from this. In contrast, both RescueTime and activity tracker data enabled participants to zoom in and out of the data, lending itself to revealing patterns. Discussions around both these data types led to comparing of these patterns, and also sharing of explanations or interpretations of them. RescueTime patterns were mainly at a daily level, whereas participants discussing activity tracker data zoomed out and looked at weekly/monthly/yearly cycles. This might be the nature of the data - that there is enough fidelity in RescueTime data to make sense of behaviour over the course of a day, whereas it makes more sense to look at activity data over larger time scales - or it could just be that our RescueTime participants only had two weeks' worth of data to look at so had to focus at that level of detail. In either case, the granularity of the data, and the timescale over which it is available, will affect the focus of participants' discussions, and therefore the outcomes of these discussions.

Familiarity with data We observed more new insights arising from looking at RescueTime data. This could be because the participants had not collected or reflected on this kind of data before (compared to participants from the other two types of datasets), and it therefore allowed them to observe things about when and how they spend their time on their computer or phone that they were not previously aware of. In contrast, the activity tracker participants and Autographer participants were familiar with their data, and had previously looked at and potentially gained such new insights from it. This suggests that the novelty of the data itself and its ability to offer participants a new perspective on their logged experience is what led to most of their gaining of new insights, rather than the process of sharing. However, there was also evidence of new insights gained in conversations around familiar data - arising from their partners suggesting a new way of interpreting the data, or our conversational prompts leading to new ways of thinking about the data. In particular statements of intentions to change came about through comparing and contrasting with others. Additionally, as the data becomes more familiar and people stop looking at it [21], noticing new things and gaining new insights just from the data is likely to decrease. Therefore, sharing and discussing data could be a useful way to prompt further reflection, especially for less novel data.

Familiarity with tools Participants shared ideas around and practices with data collection tools, particularly for supporting further understanding of the WLB topics we asked them to discuss around their data. This is akin to 'over the shoulder learning' [22] where people are found to learn from others' use of software tool in a very ad-hoc way in a working environment. When using PI tools for behaviour change, this kind of support could be particularly valuable, especially for less tech-savvy users. We 
argue that, although such technologies may offer features to support the aspects of behaviour change, there is no guarantee that people discover or correctly use these features [23].

\subsection{How knowledge led to intentions for action}

Whilst new insights from the data was one mechanism that prompted participants' intention to change - most ideas for things they could actually $d o$ to change arose from sharing routines and strategies with each other. Even though participants may have gained the knowledge (e.g., they spent too long on social media when working from home), discussing this with their partner not only allowed them to realise this was not an uncommon problem and to empathise with each otherbut to share the actions others had tried to overcome this problem (e.g., removing the Facebook app).

Many of the solutions or ideas for action generated by our participants to improve their WLB or increase levels of physical activity are similar to and extend strategies previously documented in the literature [24-26]. Arguably participants could have come up with these solutions themselves, or could have referred to more formal sources of advice without the need for sharing and discussing data. However, the conversations allowed them to discuss ideas they had not previously thought about or tried, or to discuss each other's' experience of having tried these. This led to thinking through possible ways of acting on their knowledge almost 'trying it out for size' with their partner.

Furthermore, ideas for ways to change were not always written down immediately after the conversation, or were explored in the following conversation before making it to paper. It is well documented that reflection takes time and space and needs support $[27,28]$. It seems that conversations around data offer an opportunity for that reflection, not only between researcher and participants [19] but also amongst peers. The question of whether realising and discussing ideas for change in this way is more likely to lead to action than being given official advice is a question for further research.

\subsection{Implications}

Our findings have implications for how PI tools could help people identify opportunities for change and feel motivated to try out new strategies, by facilitating and structuring meaningful face-to-face sharing around personal data, and suggest that future research should systematically explore ways of doing this. Our findings also suggest ways we could facilitate remote sharing or even prompt more effective self-reflection on data. We outline some of these ideas below. One aspect we would like to emphasise when considering how to redesign sharing experiences is around the issue of privacy: to avoid the dangers and gain the benefits of this it is important to rethink how PI tools can enable data sharing in meaningful and secure ways.
Encourage recontextualising of one's data PI tools should encourage description, explanation and questioning of data by facilitating conversation, or possibly through question prompts, storytelling, or by suggesting new interpretations of data. This differs from what others have previously suggested $[1,8,14]$ as we have highlighted how the process of recontextualising involves various steps that go beyond just explaining. Furthermore when sharing, what the data collector might think is enough information, is not necessarily what the observer does, highlighting another benefit to conversation.

Support meaningful comparing and contrasting of data By means of enriching shared aggregates of decontextualised data, PI tools should allow more accurate and deeper comparisons of contextualised data between smaller groups. This could be through matching with other relevant anonymous, annotated data streams (e.g., data-pals) or facilitating directed face-to-face or online discussion between sharers.

Highlight routines, problems, strategies and tips PI tools should help people identify common problems from their own data. Directing them towards successful strategies and tips (though booklets, app advice or other means) could trigger and motivate change, as evidenced by our data.

Create time for shared reflection Time is required to gain insight from data and contemplate ways to act on this insight. People in workplaces, community groups or schools could benefit from doing this in a social co-located situation, by making it an enjoyable experience where users retain control over how and to whom the data is shared.

\section{Conclusion}

Personal informatics technologies support the collection of and reflection on personal data, but enabling people to learn from and act on this data is still an on-going challenge. In order to explore ways of supporting people learning from and being motivated to take action on personal data, we conducted a series of data life-swap workshops on the topic of work-life balance where participants brought along and discussed with others personal data they had collected about themselves. These discussions highlight the ways in which the data is not the whole story: conversations around shared data went beyond the data, and through comparing and contrasting their experiences, and sharing strategies and tips, enabled participants to gain deeper insight into themselves and to identify and motivate action. This has implications for ways to support use of PI tools by focusing on structuring their use in social situations and beyond, to recontextualise data and add meaningful narratives to the numbers in a way that does not challenge privacy concerns and allows users to be in control of their data. 
Funding information This work was funded by the Engineering and Physical Sciences Research Council (EPSRC) through the Balance Network: Exploring Work-Life Balance in the Digital Economy EP/ K025619/2.

Open Access This article is licensed under a Creative Commons Attribution 4.0 International License, which permits use, sharing, adaptation, distribution and reproduction in any medium or format, as long as you give appropriate credit to the original author(s) and the source, provide a link to the Creative Commons licence, and indicate if changes were made. The images or other third party material in this article are included in the article's Creative Commons licence, unless indicated otherwise in a credit line to the material. If material is not included in the article's Creative Commons licence and your intended use is not permitted by statutory regulation or exceeds the permitted use, you will need to obtain permission directly from the copyright holder. To view a copy of this licence, visit http://creativecommons.org/licenses/by/4.0/.

\section{References}

1. Elsden C, Nissen B, Garbett A, et al (2016) Metadating: exploring the romance and future of personal data. In: Proceedings of the $2016 \mathrm{CHI}$ conference on human factors in computing systems. ACM, pp 685-698

2. Gartenberg C (2018) How do Apple's screen time and Google digital wellbeing stack up? In: The Verge. https://www.theverge. com/2018/6/5/17426922/apple-digital-health-vs-googlewellbeing-time-well-spent-wwdc-2018. Accessed 21 Sep 2018

3. Li I, Dey AK, Forlizzi J (2011) Understanding my data, myself: supporting self-reflection with Ubicomp technologies. In: Proceedings of the 13th international conference on ubiquitous computing. ACM, New York, NY, USA, pp 405-414

4. Fleck R, Fitzpatrick G (2009) Teachers' and tutors' social reflection around SenseCam images. Int J Human-Computer Studies 67: 1024-1036. https://doi.org/10.1016/j.ijhcs.2009.09.004

5. Ploderer B, Smith W, Howard S, et al (2012) Things you don't want to know about yourself: ambivalence about tracking and sharing personal information for behaviour change. In: Proceedings of the 24th Australian computer-human interaction conference. ACM, New York, NY, USA, pp 489-492

6. Lupton D (2014) Self-tracking cultures: towards a sociology of personal informatics. In: proceedings of the 26th Australian computer-human interaction conference on designing futures: the future of design. ACM, pp 77-86

7. Cox AL, Bird J, Fleck R (2013) Digital epiphanies: how self-knowledge can change habits and our attitudes towards them. In: Presented at: The 27th International British Computer Society Human Computer Interaction Conference: The Internet of things, Brunel University, London, UK. (2013). Brunel University, London, UK

8. Epstein DA, Jacobson BH, Bales E, et al (2015) From nobody cares to way to go!: a design framework for social sharing in personal informatics. In: proceedings of the 18 th ACM conference on Computer Supported Cooperative Work \& Social Computing. ACM, pp 1622-1636

9. Rooksby J, Rost M, Morrison A, Chalmers M (2014) Personal tracking as lived informatics. In: Proceedings of the SIGCHI conference on human factors in computing systems. ACM, New York, NY, USA, pp 1163-1172

10. Ploderer B, Reitberger W, Oinas-Kukkonen H, van Gemert-Pijnen J (2014) Social interaction and reflection for behaviour change. Pers Ubiquit Comput 18:1667-1676. https://doi.org/10.1007/s00779-0140779-y
11. Dolan P, Hallsworth M, Halpern D, et al (2010) MINDSPACE: Influencing behaviour through public policy

12. Kalnikaite V, Bird J, Rogers Y (2013) Decision-making in the aisles: informing, overwhelming or nudging supermarket shoppers? Pers Ubiquit Comput 17:1247-1259. https://doi.org/10.1007/ s00779-012-0589-z

13. Consolvo S, Everitt K, Smith I, Landay JA (2006) Design requirements for technologies that encourage physical activity. In: Proceedings of the SIGCHI conference on human factors in computing systems. ACM, New York, NY, USA, pp 457-466

14. Tolmie P, Crabtree A, Rodden T, et al (2016) "This has to be the cats": personal data legibility in networked sensing systems. In: Proceedings of the 19th ACM conference on ComputerSupported Cooperative Work \& Social Computing. ACM, New York, NY, USA, pp 491-502

15. Graham L, Tang A, Neustaedter C (2016) Help me help you: shared reflection for personal data. In: Proceedings of the 19th international conference on supporting group work. ACM, New York, NY, USA, pp 99-109

16. Skatova A, Bedwell B, Shipp V, et al (2016) The role of ICT in office work breaks. In: Proceedings of the $2016 \mathrm{CHI}$ conference on human factors in computing systems. ACM, pp 3049-3060

17. Boud D, Keogh R, Walker D (1985) Reflection: turning experience into learning. London: Kogan

18. Fleck R (2012) Rating reflection on experience: a case study of teachers' and tutors' reflection around images. Interact Comput 24:439-449. https://doi.org/10.1016/j.intcom.2012.07.003

19. Kwasnicka D, Dombrowski SU, White M, Sniehotta FF (2015) Data-prompted interviews: using individual ecological data to stimulate narratives and explore meanings. Health Psychol 34:1191

20. Ruckenstein M (2014) Visualized and interacted life: personal analytics and engagements with data doubles. Societies 4:68-84. https://doi.org/10.3390/soc4010068

21. Gouveia R, Karapanos E, Hassenzahl M (2015) How do we engage with activity trackers?: a longitudinal study of Habito. In: proceedings of the 2015 ACM international joint conference on pervasive and ubiquitous computing. ACM, pp 1305-1316

22. Twidale MB (2005) Over the shoulder learning: supporting brief informal learning. Comput Supported Coop Work 14:505-547. https://doi.org/10.1007/s10606-005-9007-7

23. Mercer K, Li M, Giangregorio L, Burns C, Grindrod K (2016) Behavior change techniques present in wearable activity trackers: a critical analysis. JMIR Mhealth Uhealth 4:e40. https://doi.org/10. 2196/mhealth.4461

24. Cecchinato M, Cox AL, Bird J (2017) Always on(line)? User experience of smartwatches and their role within multi-device ecologies. In: Proceedings of the SIGCHI Conference on Human Factors in Computing Systems. https://doi.org/10.1145/3025453.3025538. Accessed 21 Sep 2018

25. Cecchinato ME, Cox AL, Bird J (2015) Working 9-5?: professional differences in email and boundary management practices. In: Proceedings of the 33rd annual ACM conference on human factors in computing systems. ACM, New York, NY, USA, pp 3989-3998

26. Ciolfi L, Lockley E (2018) From work to life and Back again: examining the digitally-mediated work/life practices of a Group of Knowledge Workers. Computer Supported Coop Work (CSCW) 27:803-839. https://doi.org/10.1007/s10606-018-9315-3

27. Fleck R (2012) Designing for reflection, Sustainability and Simplicity

28. Moon JA (2000) Reflection in learning and professional development: theory and practice, new Ed edition. Routledge, London

Publisher's note Springer Nature remains neutral with regard to jurisdictional claims in published maps and institutional affiliations. 